\title{
Centro de Cáncer ABC, más de una década al cuidado de pacientes oncológicos en México
}

\section{ABC Cancer Center, more than a decade of Cancer Care in Mexico}

\author{
Raquel Gerson Cwilich*
}

Citar como: Gerson CR. Centro de Cáncer ABC, más de una década al cuidado de pacientes oncológicos en México. An Med ABC. 2021; 66 (4): 297-311. https://dx.doi.org/10.35366/102931

\section{RESUMEN}

El Centro de Cáncer del Hospital ABC comenzó sus operaciones en noviembre de 2009 con las especialidades de Oncología médica, radioterapia y cirugía oncológica, todas ellas en conjunto con la unidad de apoyo a pacientes y familiares. Posteriormente, una mayor inversión y un mayor enfoque en lo académico llevaron al establecimiento de programas clínico-patológicos y sesiones universitarias. La radiocirugía fue añadida en 2012 y los aceleradores lineales en 2020. Simultáneo a la adición de cubículos de quimioterapia ambulatoria, llegaron módulos de inmunoterapia y bioterapia. Una unión rigurosa entre la atención al paciente, la dedicación a la investigación y el seguimiento de la creciente incidencia de cáncer, así como el aumento de la esperanza de vida, caracteriza a todas las innovaciones del Centro. Por sí mismo, el Centro es un estándar para el rendimiento académico y la enseñanza, incluida la aprobación de cursos de nivel universitario para la subespecialidad en oncología médica. El Centro cuenta con muchos servicios: distribución farmacéutica, infusiones y transfusiones, alimentación parenteral, fisioterapia, nutrición, salud emocional y mental, y oncoimagen; todo ello unido bajo el lema del bienestar y la calidad de vida. Dos pilares fundamentales conforman el enfoque del Centro: la investigación y la docencia activa, mediante reuniones para estudios de caso. El desarrollo de proyectos de investigación ha dado lugar a muchos programas que son parte integral de la destreza científica del Centro, incluida la investigación en biología molecular, secuenciación de ADN, muestreo y cultivo celular e investigación con células madre. Los protocolos avanzan a diario con cada nuevo descubrimiento. Nuestra Unidad Médica, incluso, se ha hecho un nombre en el campo de la biopsia líquida con un enfoque progresivo en el tratamiento individualizado y diagnósticos refinados; los avances genómicos

\section{ABSTRACT}

The ABC Hospital's Cancer Center began operations in 2009 with the following subspecialties: medical oncology, radiotherapy, and oncosurgery, all tied to units dedicated to supporting patients and their loved ones. Afterwards, further investment and increased focus on academics led to the establishment of clinicopathological programs and collegiate sessions. Radiosurgery was added in 2012, and linear accelerators in 2020. Parallel to the addition of ambulatory chemotherapy cubicles came modules for immunotherapy and biotherapy. A rigorous union between attentive patient care, dedication to investigation, and tracking the rising incidence of cancer and increasing life expectancy characterizes every Center innovation. The Center itself is a standard for academic achievement and teaching, including the approval of university level courses for the subspecialty in medical oncology. The Center counts with many services: distribution of pharmaceuticals, infusions, transfusions, parenteral feeding, physiotherapy; nutrition, emotional and mental health, oncoimaging; all these joined together under the banner of wellness and life quality. Two main pillars inform the Center's approach: investigation and active teaching in mediated reunions for case studies. The development of investigative projects has birthed many programs integral to the Center's scientific prowess, including investigative molecular biology, DNA sequencing, cellular sampling and culturing, and stem cell research. Protocols advance daily with each new discovery. Our Medical Unit has even made a name for itself in the field of liquid biopsy with a progressive focus on individualized treatment and refined diagnostics; genomic breakthroughs led to the establishment of the High Specialty units and Hereditary Cancer Clinic. The Center's proactive and dynamic work focuses on precision-based
* Directora del Centro de Cáncer, Departamento de Oncología Médica.
Centro Médico ABC, Institución de Asistencia Privada (IAP), México.

Recibido: 03/06/2021. Aceptado: 16/11/2021.
Correspondencia:

Dra. Raquel Gerson Cwilich

E-mail: rgerson1@yahoo.com 
llevaron al establecimiento de las Unidades de Alta Especialidad y la Clínica de Cáncer Hereditario. El trabajo proactivo y dinámico del Centro se centra en el tratamiento de precisión, para así detectar factores de riesgo, establecer un diagnóstico precoz y manejar a los pacientes con la atención que merecen. El objetivo es convertirse en un referente de la oncología médica y ser reconocido a través de la acreditación internacional.

Palabras clave: Centro de cáncer, cuidados, cáncer en México.

Abreviaturas:

$\mathrm{CCABC}=$ Centro de Cáncer $\mathrm{ABC}$.

\section{HISTORIA}

El 22 de mayo de 2007 fue colocada la primera piedra en lo que sería el Centro de Cáncer del Centro Médico $\mathrm{ABC}$. Fue un proyecto contemplado como una necesidad básica en la atención de alta especialidad para pacientes con cáncer, para aquéllos que fueran tratados de dicha enfermedad y para quienes en su expresión fenotípica manifiestan un alto riesgo para su desarrollo.

En noviembre de 2009, arranca operaciones el Centro de Cáncer del Centro Médico ABC, con 14 cubículos de Quimioterapia ambulatoria, un tomógrafo simulador y un acelerador lineal en Radioterapia. Con esta configuración, se otorgan los tratamientos iniciales y se fueron agregando servicios y acciones importantes, destacando la apertura del «Círculo de ganadores» en el 2010 para apoyo de pacientes, familiares y sobrevivientes de cáncer.

Paralelo a lo anterior, la academia siguió un curso progresivo ascendente en un enfoque multidisciplinario, y en 2011 inicia la Cátedra Dr. Lira Puerto en memoria del reconocido oncólogo médico Víctor Lira Puerto q.e.p.d. en las sesiones ordinarias semanales, las cuales con visitantes externos y personal endogámico el número de participantes sobrepasa a más de 40 profesionales en sesiones clínico patológicas semanales.

En el año 2012, en Radioterapia arranca en operación el equipo Novalis Tx-Varian para radiocirugía y el equipo Clinac iX-Varian, lo que coloca al Departamento en la vanguardia en tratamientos de cáncer. En 2015 inician tratamientos con equipo de braquiterapia de alta tasa, tratamientos que eran administrados en instituciones que contaban con dichos equipos hasta lograr entonces contar con un equipo propio institucional. En el año 2020 se adquiere un nuevo acelerador lineal Versa HD-Elekta.

La oncología médica crecía y en el 2018 se incrementan los cubículos en quimioterapia ambulatoria a un total de 19 . treatment to thus detect risk factors, establish early diagnosis, and handle patients with the care they deserve. The goal is to become a benchmark for medical oncology and be recognised through international accreditation.

Keywords: Cancer center, cares, cancer in Mexico.

En la continuación del ascenso académico se organiza el Primer Simposio Internacional de Cáncer Colorrectal, asimismo en el 2018, 2019 y 2020 Simposio Internacional de Cáncer de Pulmón.

En sus primeras acciones, y como requisito de necesidad indispensable de crecimiento y expansión, se inaugura en 2019 el Centro de Infusión en el Campus Santa Fe.

\section{DEFINICIÓN DE UN CENTRO DE CÁNCER}

El término Centro de Cáncer adolece de una definición «fija». En algunos países como en los Estados Unidos existe un conjunto de Centros de Cáncer desarrollados con designación oficial desde el Instituto Nacional del Cáncer; sin embargo, parte de la designación se utiliza para describirlos en la discusión de las metas y funciones de Centros de Cáncer. La designación del Instituto de Cáncer se basa en esfuerzos para reducir la morbilidad y mortalidad por cáncer. Este no incluye de forma única proveer al Estado del arte del tratamiento, sino también de la investigación para la producción de nuevos conocimientos sobre la naturaleza básica del cáncer respecto a los nuevos y más efectivos acercamientos en la prevención, diagnóstico y terapéutica del cáncer.

La descripción oficial reconocida para los Centros de Cáncer refiere lo siguiente: «Los sitios donde principalmente se otorga los avances médicos a los pacientes y sus familias y los principales profesionales en cuidados para la salud».

Centro de Cáncer se refiere a instituciones únicas que se especializan sobre todo y principalmente en el diagnóstico y tratamiento de cáncer, en algunas ocasiones pueden formar un consorcio de cooperación con un programa integrado para neoplasias malignas.

Los Centros de Cáncer en otros países pueden enfatizar investigación básica, poseen los cuidados de infraestructura para el diagnóstico, el tratamiento y la prevención. Podrán ser pioneros en nuevos tratamientos, el establecimiento del estado del arte en la terapia y otros aspectos del control del Cáncer y actuar a su vez como centro de referencia en el país. 


\section{CENTRO DE CÁNCER DE EXCELENCIA ${ }^{1}$}

Un excelente centro oncológico es un recurso local, regional y nacional, que tiene un impacto que va más allá de sus propios muros en comunidades a las que sirve directamente y por el conocimiento generalizable que crea en el mundo en general.

\section{Criterios, requisitos ${ }^{2}$}

Capacidad para integrar diversos enfoques de investigación sobre el cáncer. Los criterios incluyen:

1. Fuerte investigación de laboratorio básica en campos como biología, inmunología y genética molecular.

2. La capacidad de convertir los resultados de la investigación en práctica clínica.

3. Un registro de estudios de investigación clínica innovadores en la comunidad atendida por el centro.

4. Realización de ensayos clínicos de alta prioridad para terapias de vanguardia.

5. Sólidos programas de investigación en prevención y control del cáncer.

6. Formación investigadora y educación continua para profesionales de la salud.

7. Una amplia gama de servicios de información sobre el cáncer para pacientes, profesionales y la comunidad.

8. Compromiso con los servicios comunitarios y la divulgación relacionada con la prevención y el control del cáncer.

\section{¿Qué es un centro oncológico integral?}

Para obtener una designación «integral» (la clasificación más alta otorgada por el Instituto Nacional del Cáncer), un centro debe cumplir con criterios específicos:

1. Fuerte investigación básica de laboratorio y clínica sobre el cáncer y la investigación traslacional que forma el puente entre ellos.

2. Tratamientos innovadores contra el cáncer que incluyen ensayos clínicos.

3. Programas de prevención y control del cáncer.

4. Formación y educación de los profesionales de la salud.

5. Servicios de información sobre el cáncer.

6. Difusión y educación comunitaria.

\section{La importancia de un Centro de Cáncer}

La carga del cáncer:

1. Incidencia del cáncer en aumento/mayor expectativa de vida.

2. Prevalencia mayor/eficacia de tratamientos.

3. Diagnóstico más temprano/avances en tecnología.

4. Mayor supervivencia en 50 años.

5. Aumento en $40 \%$ para 2030.

6. Tercera causa de mortalidad en México.

7. Del total de ingresos al Hospital ABC-40\% por cáncer.

8. Desafío, reto en el aumento progresivo, exige respuesta planificada.

Bases científicas centro de atención al paciente de cáncer:

1. Inminente creación de un Programa Universitario Académico.

2. Basado en pilares: cirugía, radioterapia, terapia sistémica.

3. Quimioterapia mejor conocimiento, menor toxicidad.

4. Promesa última década: tratamientos dirigidos e inmunoterapia.

5. Incorporación de la medicina de precisión, inteligencia artificial, caracterización molecular y genética del tumor, tratamiento individualizado.

\section{Curso Universitario de Subespecialización Médica en Oncología Médica del Centro de Cáncer}

Está integrado por:

1. Nueve residentes, tres egresados por año.

2. Dos profesores ante la UNAM, > 30 especialistas participantes como profesores invitados.

3. Seminario de Atención Médica.

4. Seminario de Investigación.

5. Curso de Biología Molecular.

6. Seminario de Tesis.

7. Seminarios de apoyo y casos problema.

8. Sesión General de Oncología Médica.

9. Sesiones Monográficas.

10. Seminarios para enfermería.

11. Aprobación de $100 \%$ por el PUEM (Programa Único de Especializaciones Médicas).

12. Aprobación Consejo Nacional de Oncología Médica en los últimos cinco años. 
13. Presencia nacional e internacional en eventos médicos académicos.

14. Publicaciones y carteles.

\section{Funciones Centro de Cáncer}

Las funciones centrales que se han creado en el Centro de Cáncer $\mathrm{ABC}$ son las siguientes:

1. Cuidados del paciente: incluyen los servicios de oncología médica, radioterapia cirugía, imagen, e imagen molecular, patología, servicio psicosocial, terapia paliativa y servicios de apoyo, central de mezclas e infusiones.

2. Entrenamiento en todas las funciones disponibles para el Centro de Cáncer de acuerdo a las necesidades y capacidades de la institución y del país.

3. Investigación enfocada a cuestiones clínicas de importancia local y particular.

4. Detección del cáncer y programas de prevención.

5. Extensión comunitaria, que incluye educación, programas de prevención y cuidados paliativos de la comunidad.

6. Vigilancia y monitoreo de factores de riesgo relevantes y datos específicos de cáncer consistentes con programas nacionales.

7. Comunicación y tecnología de la información, con propósitos de conexiones con el país, así como en forma internacional.

Para esto, el Centro de Cáncer ABC cuenta con el departamento de oncología médica, que provee cuidado clínico y conduce la investigación transformativa básica. ${ }^{1}$

\section{CENTRO ONCOLÓGICO INTEGRAL ${ }^{3}$}

La mayoría de los países y numerosas organizaciones globales y locales están abordando los desafíos del cáncer incluido el desarrollo de programas nacionales integrales de control del cáncer, diseñados para reducir el número de casos y muertes por cáncer y mejorar la calidad de vida de los pacientes a través de estrategias basadas en evidencia para la prevención, detección temprana, diagnóstico, tratamiento y paliación. Un programa nacional de control del cáncer aborda las funciones y la entrega de varios componentes en el control del cáncer.

\section{Componentes del sistema de cáncer}

La Organización Mundial de la Salud OMS recomienda que todas las naciones tengan un plan de control del cáncer que incluya estos componentes: prevención, detección, diagnóstico, tratamiento, supervivencia y cuidados paliativos al final de la vida (Figura 1).

\section{Departamento de Oncología Médica}

El Departamento de Oncología Médica provee atención clínica compasionada a pacientes con Cáncer, conduce investigación clínica, básica, transformativa, traslacional y aporta educación de punta, entrenamiento y tutorial en Oncología Médica.

Sigue el concepto de división clínica e incluye hospitalización, quimioterapia ambulatoria y la consulta particular en el peldaño de la investigación traslacional. Tiene como fundamento el manejo multidisciplinario bajo guías internacionales de tratamiento. Se presentan y discuten casos «Tumor Board», así como reuniones colegiadas internacionales de medicina de precisión para arribar en el mejor manejo, un tratamiento individualizado siempre en apego a los dictados de normas internacionales.

\section{Central de mezclas}

En la central de mezclas bajo la dirección de la Farmacobióloga Sandra Palacios, antes de la creación del Centro de Cáncer, ya se atendía a un importante número de pacientes de la especialidad de oncología y hematología en el Centro Médico ABC.

El Servicio de Farmacia del Centro Médico ABC es pionero en la dispensación de mezclas estériles de medicamentos, su existencia data desde 1990.

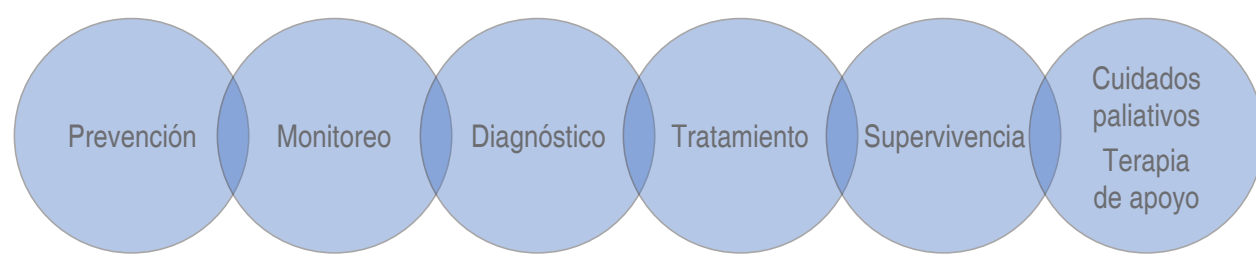

Recomendación de la Organización Mundial de la Salud a todas las naciones tener Plan de Control de Cáncer
Figura 1:

Plan de Control de Cáncer. 


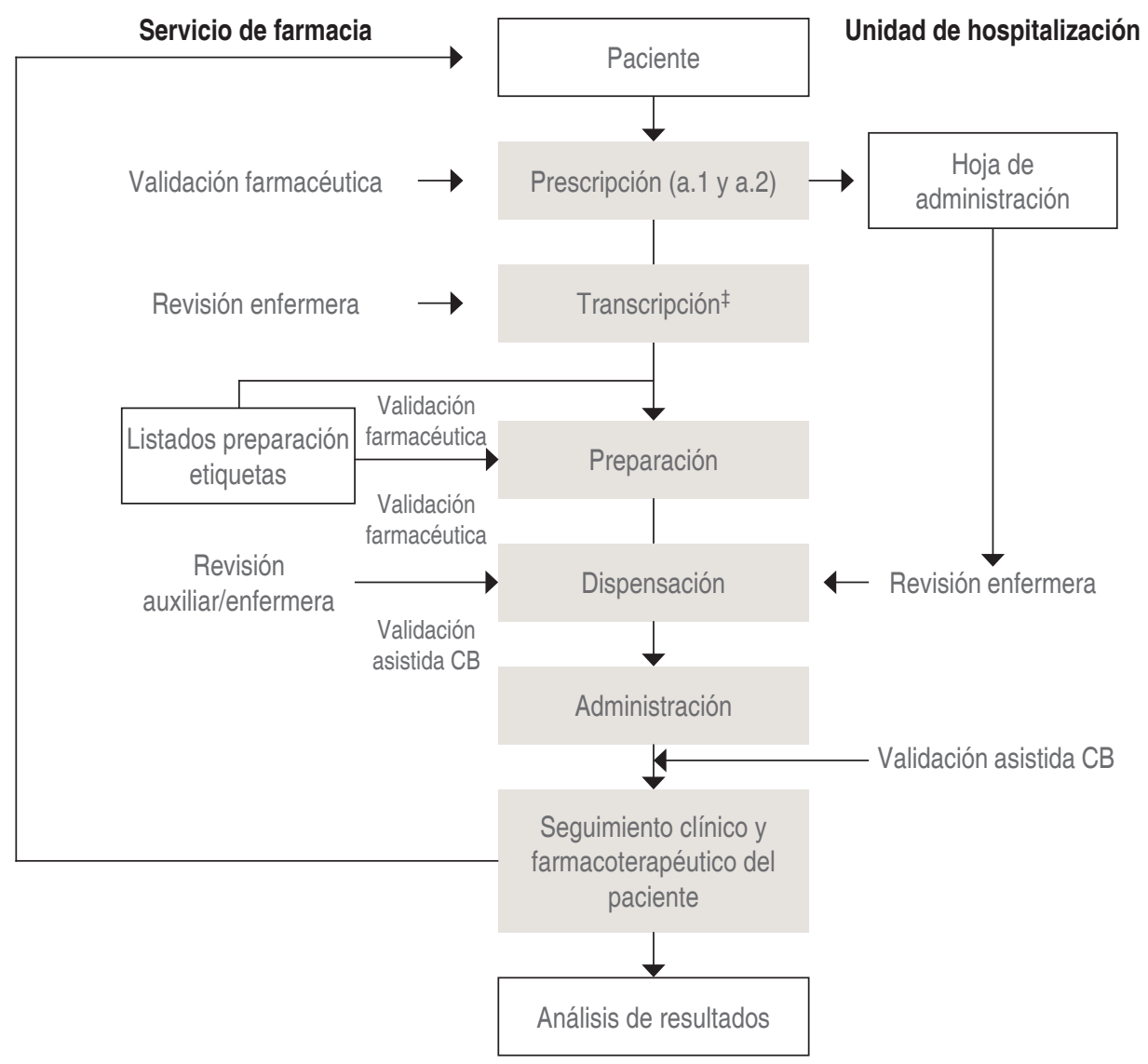

Figura 2:

Proceso farmacoterapéutico.

a.1) Prescripción manual.

a.2) Prescripción electrónica;

¥Se obvia en la prescripción electrónica.

$\mathrm{CB}=$ Código de barras.

La labor de un centro de mezclas es realizar la dispensación de las mezclas estériles, para el apoyo al nuevo centro de quimioterapia ambulatoria del Centro de Cáncer se consideró la creación de un cuarto adicional exclusivo para la preparación de las mezclas de agentes citotóxicos, equipado con un gabinete de seguridad biológica que proporcionara un ambiente seguro para el personal y su respectiva antesala.

Sin embargo, además de contar con la capacidad instalada, había que insistir en la formación del personal y contar con los recursos informáticos, ${ }^{4}$ además de estandarizar las soluciones en las que se debe preparar cada fármaco para asegurar compatibilidad y obtener estabilidad de los fármacos antineoplásicos y de soporte.

Se seleccionó la herramienta que aún se continúa utilizando en España y otros países de Iberoamérica, ${ }^{5}$ la cual funciona con el modelo de estandarización propuesto por autores españoles para evitar y prevenir errores de medicación en el proceso farmacoterapéutico colaborativo ${ }^{6}$ actualmente aplicado en instituciones nacionales (Figura 2). Realiza cálculos automáticos para evitar errores de medicación y retrasos en el total de las prescripciones hacia finales de 2013 y $100 \%$ en 2014 para el centro de quimioterapia ambulatoria.

Con apoyo del patronato del Hospital ABC y del equipo multidisciplinario, se obtuvo la primera licencia sanitaria a fines de 2014 para un centro de mezclas oncológicas en un hospital privado.

\section{RESULTADOS}

Una vez confirmados los tratamientos mediante esta sistematización, los resultados se reflejaron en los tiempos de disponibilidad (Figura 3). En la actualidad se conserva de manera sostenida desde la confirmación del tratamiento a su disponibilidad el promedio entre 37-40 minutos.

En su historial, la Central de Mezclas del Centro de Cáncer ha preparado desde enero de 2014 hasta 16 de marzo 2020 52,816 preparaciones para el Servicio de Quimioterapia ambulatoria, $82 \%$ de éstas corresponden a tratamiento antineoplásico y que a su vez corresponden a $47 \%$ de las preparaciones totales 
( $\mathrm{n}=111,610)$, ya que se suman el Servicio de hospitalización, Oncopediatría, Unidad de Trasplantes y Centro de Infusión Santa Fe.

Los pacientes y prescripciones para los tratamientos antineoplásicos y de soporte dispensados por la central de mezclas del Centro de Cáncer se han mantenido de manera constante como se observa en la Figura 4.

De las preparaciones realizadas para el Centro de Cáncer, en el periodo enero 2014 a marzo 2020, la central de mezclas ha atendido 2,715 pacientes con diagnósticos oncológico-hematológicos, esto representa $76 \%$ del total, $24 \%$ restante corresponde a diversos diagnósticos: anemia ferropriva, enfermedades autoinmunes y neurodegenerativas.

Los principales diagnósticos oncológicos son: cáncer de mama $33.6 \%$, cáncer de ovario $7.3 \%$, cáncer colorrectal $7.1 \%$, linfomas $6.7 \%$, cáncer de pulmón $6.2 \%$, páncreas $5.2 \%$ (Figura 5).

Es importante mencionar la presencia de la unidad de calidad, la cual realiza la verificación de la prescripción médica y lleva a cabo actividades de farmacovigilancia. El responsable sanitario es vigilante de que se efectúen cada uno de los procedimientos normalizados de operación y bajo Manual de Calidad; entre otras funciones principales, debe ser los «ojos de Cofepris» en la central de mezclas.

La central de mezclas del Centro de Cáncer tiene como retos y metas en su perspectiva:

1. Implantar control electrónico asistido por lector de código de barras, para completar proceso farmacoterapéutico y control de calidad cuantitativo de las preparaciones mediante control gravimétrico y cualitativo.
2. Farmacovigilancia activa en conjunto con los miembros del equipo de salud, del paciente y familiares

3. Participar en la educación al paciente que recibirá tratamiento antineoplásico por primera vez, «un paciente informado participa activamente en su propia seguridad».

En la central de mezclas del Centro de Cáncer aprendimos la importancia del Trabajo en equipo, la enseñanza enmarca la vocación de este servicio farmacéutico: colabora en el diplomado de enfermería, participa en reuniones de formación para médicos residentes de oncología médica desde el 2013 con aspectos prácticos de la terapia antineoplásica.

\section{La investigación en el centro}

La investigación traslacional persigue la mejor atención al paciente y adelantos en tratamientos para el cáncer, como respuesta a esta imperiosa necesidad, se crea el Departamento de Biología Molecular dentro del Servicio de Patología del Hospital ABC, bajo la asesoría de investigadores del Instituto de Ciencias Biomédicas de la UNAM se promueve la incorporación del Dr. Alejandro Zentella como biólogo molecular para integrar el Servicio de Biología molecular del Centro de Cáncer, quien promueve introducir a los residentes en la cátedra de biología molecular y paralelamente estimular la generación de trabajos y estudios de investigación y en alianza con el Instituto Nacional de Nutrición y Ciencias Médicas «Salvador Zubirán» y el Instituto de Investigaciones Biomédicas de la UNAM, como origen de ello se crea la primera línea de investigación con la tesis de maes-

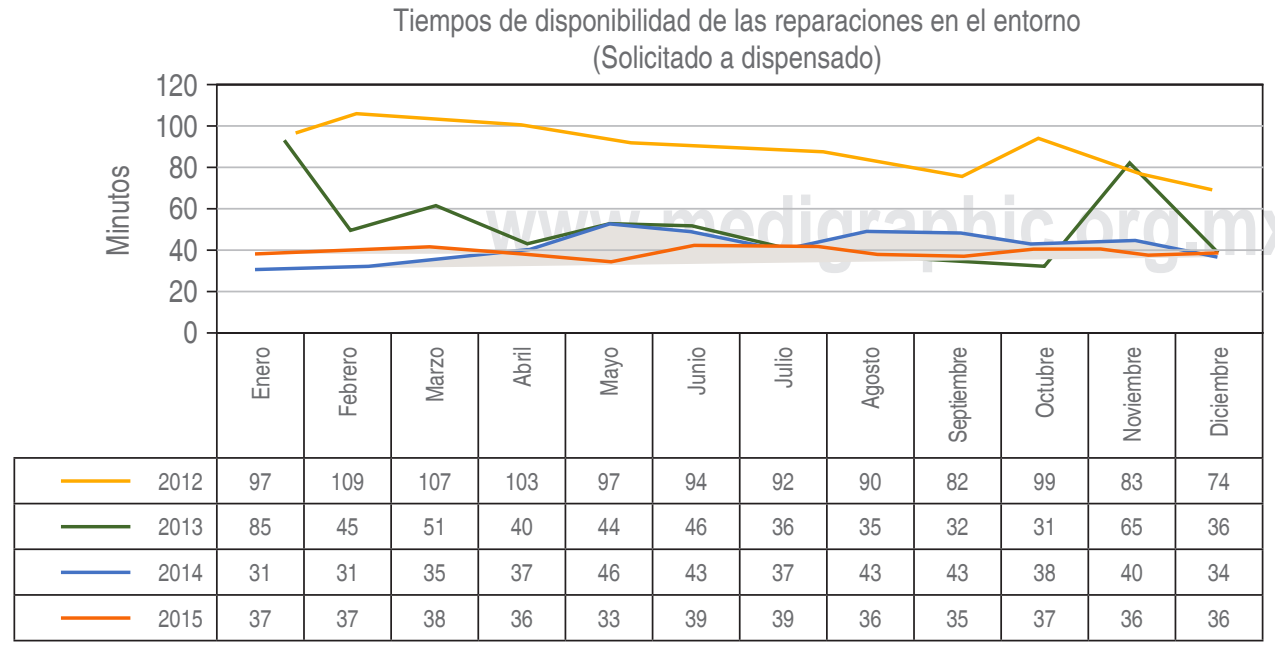

Figura 3:

Gráfico del tiempo promedio de dispensación. Gráfico del tiempo promedio de dispensación en 2012 fue de $93 \pm 10.4$ minutos, en 2013 de $45.5 \pm 15.6$ minutos, en 2014 de $38 \pm$ 4.93 minutos y en 2015 de $37 \pm 1.68$ minutos. Elaboración propia con base de datos Central de Mezclas del Centro Médico ABC y sistema Farmis-Oncofarm ${ }^{18}$ a partir de 2014. 


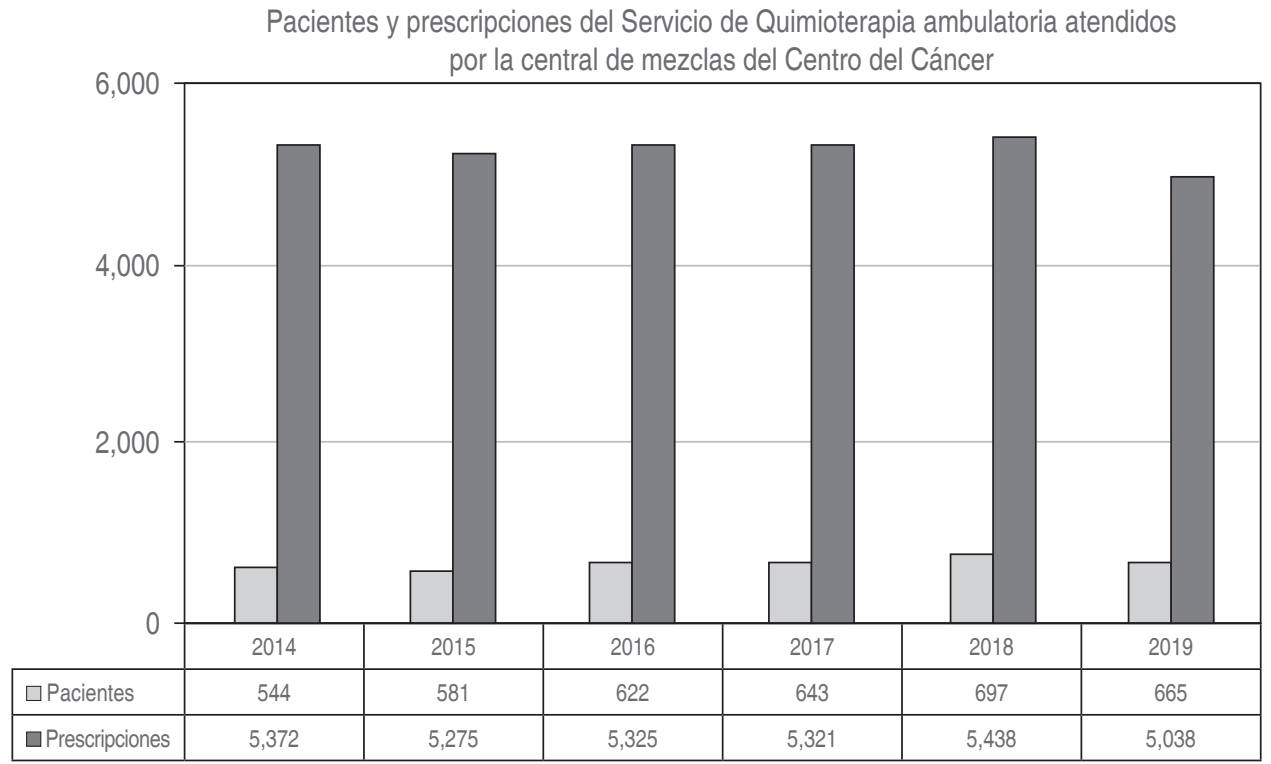

Figura 4:

Gráfico que muestra el volumen de pacientes y prescripciones totales generadas en el sistema de prescripción electrónica Farmis-Oncofarm ${ }^{\circledR}{ }^{18}$ tría de la Universidad Anáhuac de la entonces residente en medicina interna Daniela Shveid Gerson bajo el título de Cultivo Celular en Pacientes obesas y no obesas con cáncer de mama luminal A. De dicho principio han surgido varias líneas de investigación originales, con las que el centro ha participado en diferentes foros nacionales e internacionales, así como publicaciones de revistas de alto impacto. Una de las importantes adquisiciones para complementar el proyecto de la investigación traslacional en cáncer en enero de 2020 ha sido la apertura del laboratorio de investigación traslacional para poder hacer los propios cultivos celulares, bajo la guía del Dr. Zentella y la participación de todos los residentes con la mira de lograr cultivos celulares desde su inicio, con aspiraciones futuras de lograr cultivar tejidos y en algún momento el poder alcanzar secuenciación de nueva generación.

\section{Programa integral de Investigación del Centro de Cáncer A cargo del biólogo molecular Alejandro Zentella.}

El 29 de enero de 2020 se inauguró el laboratorio de investigación, que cuenta con instalaciones y equipamiento para realizar el análisis bioquímico de proteínas, así como para el cultivo de células y el estudio de sus características biológicas.

Este laboratorio depende de la dirección de investigación y cuenta con la colaboración del Instituto Nacional de Nutrición y Ciencias Médicas «Salvador Zubirán», así como del Instituto de Investigaciones
Biomédicas de la UNAM. Esta unidad complementa al laboratorio de biología molecular dedicado al estudio de ácidos nucleicos.

Vivimos un momento histórico en el que la investigación biomédica impacta en la medicina como nunca antes. Los avances en el conocimiento de las estructuras básicas de las células y sus funciones en la fisiología celular han llevado a un mejor entendimiento de las bases moleculares y genéticas de trastornos como la diabetes, cáncer o el Alzheimer. Otras enfermedades aún carecen de una comprensión equivalente como la hipertensión o la demencia. Sin importar nuestro nivel de comprensión, hoy entendemos la enfermedad como el resultado de la interacción entre versiones particulares de las proteínas codificadas en nuestros genes, el medio ambiente y nuestro estilo de vida.

La posibilidad de analizar el genoma y el comportamiento de células derivadas de un paciente particular han desarrollado los conceptos de medicina personalizada y, más aún, el de medicina de precisión. Algunas preguntas no las puede resolver el estudio de las secuencias de DNA, en este caso la posibilidad de cultivar células de los pacientes permite hacer comparaciones funcionales bajo condiciones controladas. Así iniciamos un protocolo comparando el comportamiento biológico de células de cáncer de mama luminal A derivadas de una paciente eutrófica o de una con obesidad, encontrando diferencias en su potencial de proliferación, motilidad y capacidad tumorigénica. Ahora se puede sistematizar este estudio para evaluar si estas diferencias entre estas dos 
pacientes pueden generalizarse, lo que abonaría a un pronóstico y seguimiento personalizado. Este tipo de estudios comunes en oncología traslacional pueden extenderse a otras áreas de la medicina.

Teniendo la posibilidad de establecer cultivos de células tumorales, hemos empleado derrames pleurales o líquido de ascitis malignos en pacientes con más de tres recurrencias, estos cultivos han permitido montar un ensayo de antiquimiograma que genera resultados en dos semanas y permite apoyar al oncólogo en la elección de la quimioterapia más efectiva.

A futuro nos proponemos establecer cultivos primarios derivados del tumor sólido, empleando sistemas de disgregación enzimática y mecánica de las células tumorales para cultivarlas y aplicar el mismo protocolo que hemos implementado para el antiquimiograma.

Este mismo protocolo nos dará acceso a las poblaciones de células del sistema inmune que emplearemos para implementar protocolos orientados a la aplicación de inmunoterapia.

Por supuesto que el cultivo de células derivadas de un paciente también ofrece la posibilidad de cultivar células troncales y, con ello, se abre la posibilidad de realizar protocolos de terapia celular en el campo de la medicina regenerativa, en particular las células troncales mesenquimales que se pueden derivar de la médula ósea, de la pulpa dental, del cordón umbilical o del tejido adiposo.
La naturaleza inmunomoduladora de estas células troncales mesenquimales puede emplearse para mitigar y controlar respuestas inflamatorias sistémicas, como las que se presentan en pacientes terminales con COVID-19. Cabe resaltar que la naturaleza de nuestras instalaciones la convierten en uno de los pocos laboratorios de cultivo celular que cumplen con los estándares internacionales para cultivar células humanas con fines de dar terapia celular.

\section{Clínica de cáncer hereditario}

Desde 2018 bajo la dirección de la Dra. María Teresa Toussie y asesoría genética de la Dra. Lenny Gallardo.

Con la visión de la participación genética en el origen de las neoplasias y con gran afán de ofrecer a los pacientes una atención integral en su prevención y atención, se crea la clínica de cáncer hereditario.

Se basa en servicios de consulta para personas con antecedentes personales o familiares de cáncer, bajo un enfoque multidisciplinario y de equipo para lograr brindar atención de la más alta calidad.

El Programa de Cáncer Familiar está planeado para ayudar a identificar a las personas con mayor riesgo de cáncer debido a factores hereditarios y ayudarles a comprender este riesgo.

Los aspectos clave del programa son la detección de los casos en riesgo, evaluación, educación y asesoramiento sobre el riesgo de cáncer, realizar prueba molecular de predisposición cuando sea apro-

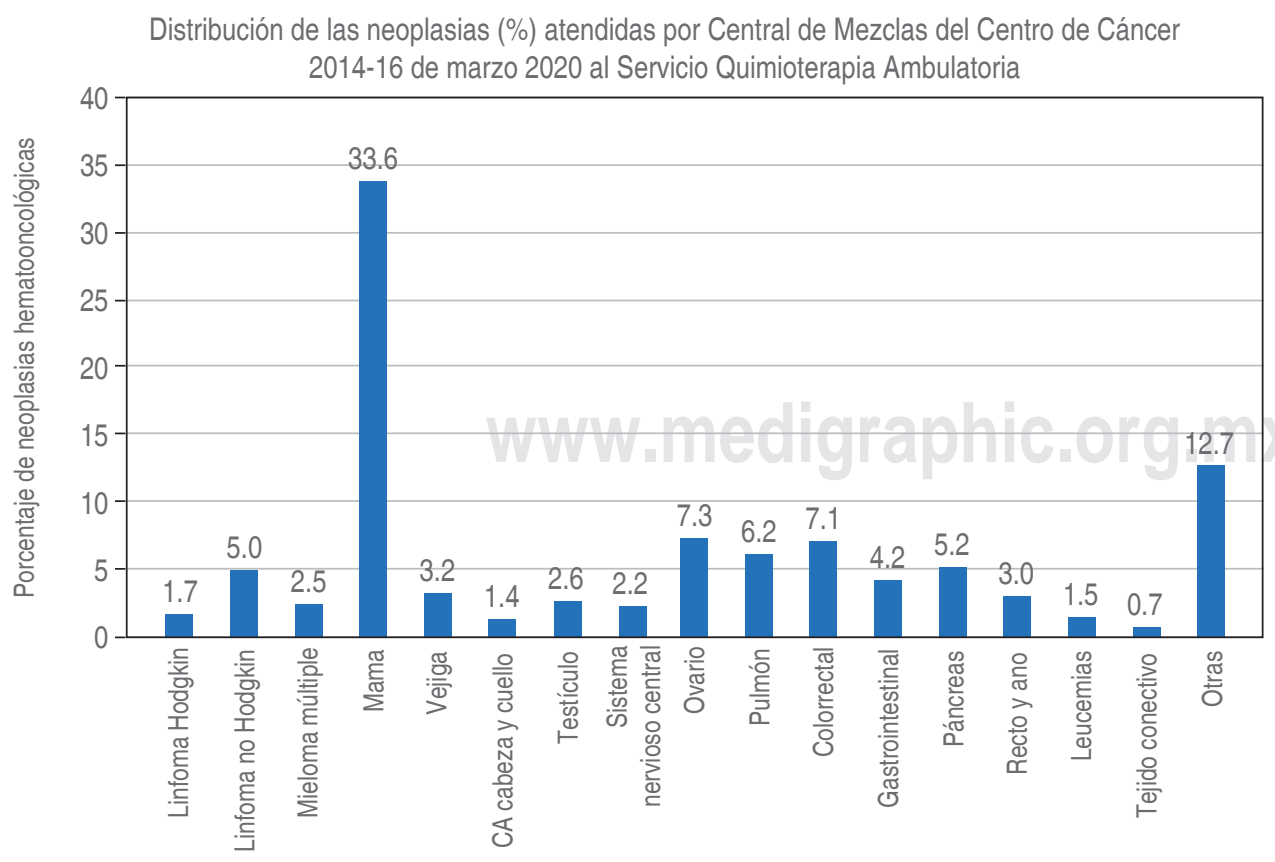

Figura 5:

Distribución de los diagnósticos atendidos por la Central de Mezclas del Centro de Cáncer en la Unidad de Quimioterapia Ambulatoria. 

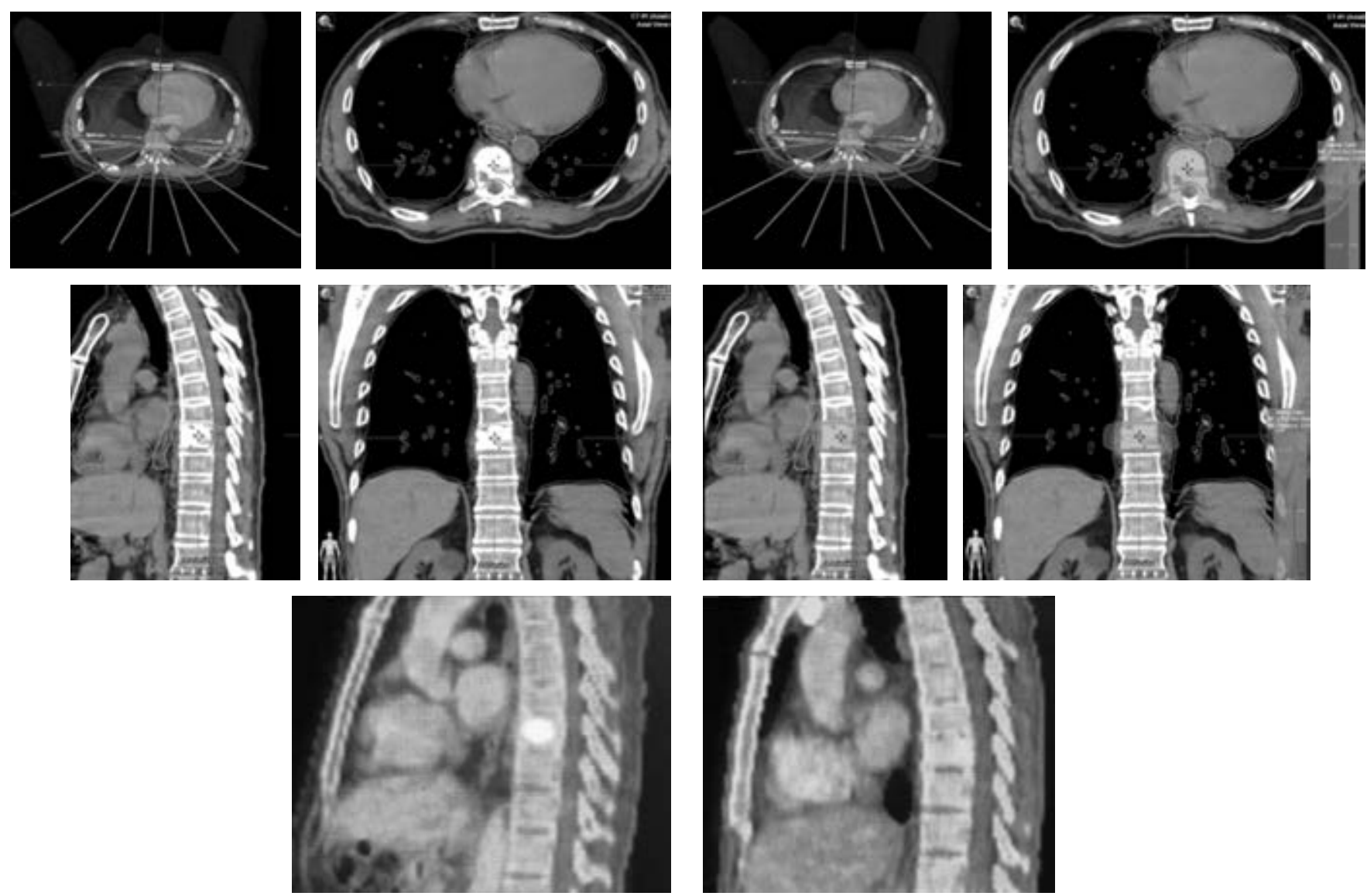

Figura 6: Paciente con metástasis espinal en T9 tratado con radiocirugía en dosis única. Se muestra la tomografía con y sin curvas de isodosis y el PET pre- y postradiocirugía izquierda y derecha respectivamente.

piado y después de esta evaluación inicial proponer planes personalizados enfocados en detección eficaz y opciones de prevención como terapias reductoras de riesgo.

Utiliza la información más actualizada sobre cáncer hereditario para la evaluación previa como para la decisión de intervenciones, que pueden ir desde un seguimiento intensivo hasta la propuesta de terapias reductoras de riesgo.

El objetivo principal es brindar atención individualizada. Los miembros del equipo son médicos genetistas con formación especializada en análisis de riesgo para cáncer hereditario, además de especialistas en biología molecular y medicina genómica.

\section{Tratamientos para el cáncer, más allá de la quimioterapia ${ }^{7}$}

Tratamiento personalizado: cada día conocemos más y mejor los mecanismos moleculares que conducen a la inducción y posterior desarrollo del cáncer, así como a las moléculas que están implicadas en este proceso. La medicina personalizada, también llamada medicina de precisión, trata de anular la actividad de estas moléculas, con lo que se impide el desarrollo del tumor. En realidad, lo que se busca es administrar el fármaco adecuado a la dosis precisa al paciente concreto, para ello, es fundamental conocer el estado mutacional de su tumor.

La medicina ultrapersonalizada consiste en adaptar el tratamiento correcto a las características individuales de cada persona. La nueva «ultrapersonalización» suma a la ecuación el nombre y apellidos de los tumores por sus alteraciones moleculares, la información acerca del estilo de vida y los hábitos de los pacientes, el género o el origen étnico. Con todos estos datos, se obtiene un retrato más acertado de cada caso y se pueden seleccionar los pacientes que más beneficio pueden obtener de un tratamiento de diseño individual. 
Biopsia líquida: por otra parte, a nivel de laboratorio, los avances en biología molecular y genética están cambiando todo el panorama. Las técnicas de secuenciación de genes y la biopsia líquida están permitiendo la identificación de alteraciones moleculares responsables de que los tumores nazcan, crezcan y se diseminen, constituyendo además dianas terapéuticas para nuevos fármacos dirigidos; estos fármacos son, en general, más eficaces y menos tóxicos, en el concepto de la oncología personalizada.

El término biopsia líquida hace referencia a que con un simple análisis de sangre se podrá detectar si existen células tumorales circulantes en la sangre, las alteraciones moleculares que presenta el paciente, medido a través del DNA circulante ctDNA, o vesículas como los exosomas. La técnica podría revolucionar el diagnóstico precoz y el tratamiento del cáncer, así como poner fin a las dolorosas e incómodas biopsias para analizar el tumor; lo cual permite la posibilidad de realizar biopsias líquidas en forma periódica que faciliten la evaluación del estado de la enfermedad, así como elección del mejor tratamiento en cada momento.

\section{Unidad de medicina de precisión en oncología}

Bajo la dirección de la Dra. Ruby Delgado reconocida Patóloga emigrada del Hospital Memorial de Nueva York (Memorial Sloan Kettering Hospital).

Los retos que surgen a partir de la oncología basada en la genómica han hecho necesaria la creación de una nueva disciplina en la oncología: Precision Oncology Decision Support o Sistemas de Apoyo para la Oncología de Precisión.

La Unidad de Medicina de Precisión en Oncología, entre otras tareas, desarrolla esta nueva disci- plina en el Centro de Cáncer ABC (CCABC). Persigue apoyar y formalizar la implementación de la oncogénica en el CCABC, y profundizar la experiencia en la misma.

Se han fortalecido alianzas con las principales compañías genómicas de secuenciación de nueva generación: Foundation Medicine, CARIS, Guardant Health, entre otras. A su vez, se han logrado interacciones directas con sus propios expertos, como ejemplo, a través del Molecular Tumor Board bimestral que se lleva a cabo con estricta periodicidad y adherencia de médicos de base y residentes entre el CCABC, y Foundation Medicine, que orienta hacia los hallazgos genómicos, el posible manejo conductual y los protocolos internacionales prevalentes al respecto.

A la vez, se están buscando pruebas innovadoras basadas en el DNA circulante que faciliten el monitoreo molecular tumor-específico y el micromanejo dinámico y predictivo del cáncer, así como pruebas basadas en el DNA germinal, para intensificar la detección del cáncer hereditario por una parte, y para refinar el tratamiento oncológico personalizado mediante la farmacogenética.

También se ha iniciado la recolección y estructuración de los resultados de las pruebas de perfil molecular tumoral, permitiendo descubrir el paisaje mutacional de los distintos tipos de tumores tratados en el CCABC, para contribuir a la ayuda en concretar el Real World Data y para armar el Real World Evidence de la oncología de precisión en el CCABC.

Y más allá de la oncogenómica, se delinean proyectos innovadores que conciben a la oncología de precisión como la caracterización y análisis multidimensional del cáncer, es decir, la ciencia de los datos del cáncer Cancer Data Science.
Prerradiocirugía

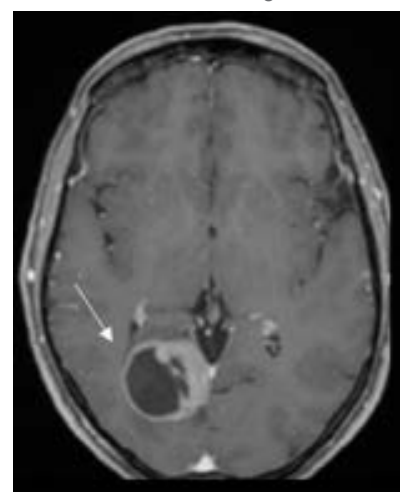

3 meses

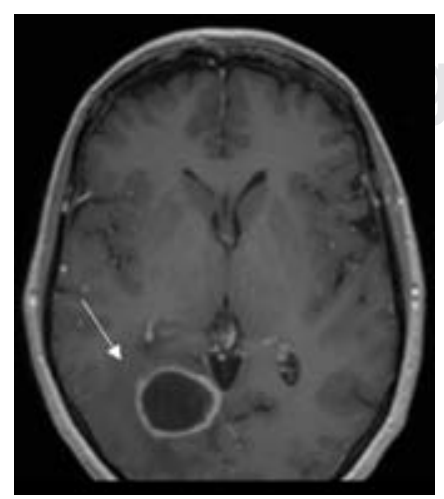

6 meses

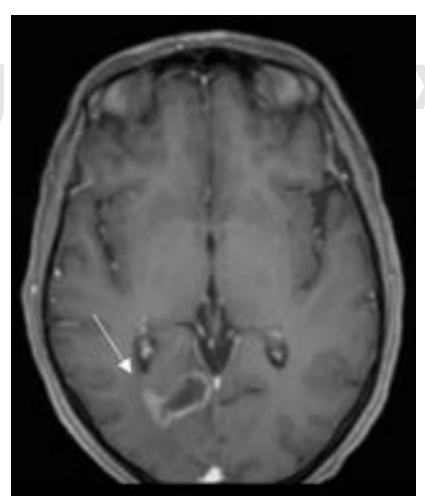

Figura 7:

Paciente femenino con adenocarcinoma de pulmón con tres metástasis tratadas con radiocirugía en dosis única. En la imagen se observa una resonancia magnética de cráneo en corte axial SPGR con contraste antes del tratamiento, a los tres y seis meses. 


\section{Servicios que integran el Centro Médico ABC}

Un peldaño importante es el Servicio de Radioterapia a cargo de la Dra. Dolores de la Mata que además de la participación central de asistencia cubre actividad académica y significativa.

1. Centro de capacitación de físicos médicos.

2. Cursos anuales nacionales e internacionales de radioterapia y radiocirugía.

3. Proyectos de investigación.

4. Participación en congresos nacionales e internacionales (SOMERA, ESTRO, ASTRO).

5. Sesiones semanales con residentes.

6. Residentes de radioterapia, ocho a 12 por año, y residentes de oncología médica del Centro Médico $\mathrm{ABC}$.

7. Sesiones desde hace más de 10 años con el Houston Methodist.

8. Sesiones internas ABC entre pares, incluyen todos los casos clínicos tratados de radioterapia a 10 años de desarrollo.

9. Sesiones con diversos servicios de la institución, neurología, hematología, pediatría y otros.

\section{Unidad de radioneurocirugía Bajo la dirección del Dr. Sergio Moreno Jiménez.}

La neurocirugía estereotáctica y la radioterapia se combinaron para desarrollar la radiocirugía, la cual consiste en la administración precisa de radiación ionizante. El primero en describir este procedimiento en 1951 fue el Prof. Lars Leksell, neurocirujano sueco. Los primeros intentos se realizaron con un sistema de estereotaxia desarrollado por Leksell y adaptando un equipo de ortovoltaje. Sin embargo, posteriormente utilizaron fuentes de Cobalto 60, lo que permitió el nacimiento del primer equipo dedicado para radiocirugía llamado Gamma Knife. A partir de ese momento, la radiocirugía ha continuado avanzando y mejorando, logrando dar tratamientos en la actualidad con rayos gamma, rayos $\mathrm{X}$ de alta energía o partículas pesadas. ${ }^{8}$

La radiocirugía es un procedimiento que requiere imágenes de alta definición para visualización del blanco a radiar, un sistema de referencias tridimensional con fines de localización, planeación y tratamiento, un sistema de planeación para realizar y optimizar el plan de tratamiento, un equipo dedicado a radiocirugía que permita tener una precisión submilimétrica bajo condiciones clínicas, y un equipo adecuado de protección, además de un equipo multidisci- plinario conformado por neurocirujanos, radiooncólogos y físicos médicos entrenados en radiocirugía. ${ }^{9}$

En México existen hospitales públicos y privados que realizan radiocirugía con muy buenos resultados; ${ }^{10}$ sin embargo, el Centro Médico ABC fue el primero en iniciar un programa de radiocirugía extracraneal incluyendo columna, pero también otros órganos como pulmón, hígado y próstata, entre otros (Figura 6).

Después de un largo esfuerzo conjunto entre el Centro Neurológico y el Centro de Cáncer del Centro Médico ABC, se logró adquirir un equipo de última generación para dar tratamientos con radiocirugía tanto craneal como extracraneal. El equipo es un acelerador lineal de 6 MV Novalis Tx-Varian (Brainlab, GmbH Germany) con sistema de colimación micromultihojas y de conos. Se logró conjuntar un equipo multidisciplinario conformado por físicos médicos, radiooncólogos y neurocirujanos, todos con una formación sólida en el campo de la radiocirugía y con experiencia previa.

El primer tratamiento se realizó el 12 de noviembre del 2012 a una paciente con una metástasis cerebral por cáncer de mama con un buen control de la enfermedad local.

Desde el inicio de su funcionamiento y hasta el 28 de junio de 2019 se habían tratado un total de 867 casos. De éstos, 451 (52\%) fueron tratados con radioterapia, 286 (33\%) con radiocirugía craneal y 130 (15\%) con radiocirugía extracraneal; cada año hemos logrado tener un mayor número de tratamientos. Las patologías más frecuentemente atendidas fueron: neoplasias 820 (95\%), vascular $37(4 \%)$ y funcional nueve (1\%). De la patología neoplásica 689 (80\%) fueron malignos y 131 (15\%) benignos considerando el total de tratamientos. En la Figura 7 se observan ejemplos de pacientes tratados en la Unidad de Radiocirugía del Centro Médico ABC.

La Clínica de Radiocirugía trabaja de una forma multidisciplinaria y colegiada, de tal manera que todos los casos son presentados en una sesión para tomar la mejor decisión posible.

\section{Atención personalizada para pacientes oncológicos Clínica Calidad de Vida bajo la dirección de Lic. Isabel Aloi.}

Se caracteriza por ser un modelo de atención integral para pacientes con cáncer, con especialistas en distintas disciplinas terapéuticas. Se brinda una atención personalizada para el paciente oncológico y sus familiares, que permita desarrollar una mejor calidad de vida, y cubren diversos aspectos de manera integral. 
1. Fisioterapia oncológica: la fisioterapia aplicada cumple con una función complementaria a todos los tratamientos médico-quirúrgicos, con la finalidad de que el paciente recupere su funcionalidad y calidad de vida, además previene futuras complicaciones.

2. Nutrición: educar y concientizar a los pacientes sobre los beneficios de llevar una buena alimentación para hacer frente a las secuelas de cáncer y sus tratamientos.

3. Salud emocional oncológica: el estrés físico y emocional que el diagnóstico de cáncer genera en el paciente y en sus familiares puede aminorarse y aprender a gestionarlo a través de la psicoterapia, logrando equilibro en sus emociones y bienestar personal; el manejo de las emociones y temores después del diagnóstico es básico en el curso del tratamiento y del seguimiento.

4. Oncoimagen: enfrentarse al cáncer es un proceso muy difícil, varios escenarios se pierden en el camino, algunos que golpean más allá de la salud. La oncoimagen es gran aliada para la recuperación de pacientes oncológicos, fortalece la autoestima, proyecta seguridad para mejorar el desenvolvimiento personal y logra mejorar la convivencia en el entorno familiar, social y laboral.

\section{GRUPO DE APOYO A PACIENTES DE CÁNCER Y SUS FAMILIARES \\ «Círculo de ganadores» Bajo la dirección de la Arq. Miriam Fastag.}

Círculo de Ganadores se fundó en junio de 2000 por iniciativa de Mario Fastag, un paciente de cáncer y su esposa Miriam, apoyados por un grupo de Damas Voluntarias del Centro Médico ABC. Inició en busca de un grupo de apoyo en México en donde los enfermos de cáncer y sus familiares pudieran encontrar apoyo emocional, lograr enfrentar los retos que planteaba el cáncer, así como compartir experiencias con otras personas, vivencias que habían tenido y deseaban extender de los grupos de apoyo a los que asistieron durante su tratamiento en el extranjero.

Los fundadores recurrieron al American Cancer Society para recibir asesoría e iniciar el proyecto. El hospital autorizó el funcionamiento del grupo, apoyado por médicos que creían en dicha iniciativa, se utilizó la Biblioteca de las Damas voluntarias para las reuniones, al inaugurarse el edificio del Centro de Cáncer, el grupo fue reinstalado. Las sesiones se llevan a cabo en forma bisemanal de forma gratuita con apertura a pacientes de todas las instituciones, para todo tipo de cáncer.

Las reuniones son escenarios variados, se enfocan en ayuda mutua, o se imparten pláticas con médicos y especialistas invitados, un taller para el bienestar de los asistentes, en ocasiones talleres de teatro, escritura, fotografía, arte y baile, también se han realizado excursiones y visitas culturales.

Como complemento se presenta una sesión de meditación previa a cada una de las reuniones, se ofrecen clases de yoga. Dos veces al año, Círculo de Ganadores hace una fiesta para diversión y esparcimiento de los asistentes y sus invitados.

En 2010, al celebrar el X Aniversario de su fundación, se publicó un libro conmemorativo titulado «Cuando nuestra vida cambia...», con 32 testimonios de pacientes y familiares.

En 2021, Círculo de Ganadores celebrará el XXI Aniversario de su inicio, donde se presentará una pieza llamada arte-objeto, en este proyecto participaron 40 personas, para ello, se impartieron tres talleres de arte dirigidos a participar y compartir experiencias para superar miedos, enojos y aceptar sentimientos.

Recibir apoyo de pacientes y familiares favorece el sentirse comprendidos por otros en situación similar, esto ayuda a enfrentar con actitud positiva los retos de la enfermedad, adquirir mayor responsabilidad frente a los tratamientos, ser paciente comprometido y activo en el cuidado de su salud y mirar cómo otros pueden superar el cáncer.

$\mathrm{Al}$ final los pacientes aprenden a retomar el control de su vida después del diagnóstico y de la etapa de los tratamientos y con orgullo se convierten en sobrevivientes.

\section{Cuidados paliativos \\ Bajo la dirección de la Dra. Margarita Araujo.}

Durante los últimos 10 años, se ha observado una prolongación de la vida con diferentes tratamientos cada vez mejor tolerados y con menos efectos secundarios, con curación o recurrencias controladas, que hacen ver a la enfermedad oncológica como un padecimiento crónico.

El marco normativo que rige el desarrollo de políticas de salud, basadas en los derechos humanos, incluye los cuidados paliativos como herramienta para afrontar la experiencia personal y social en el manejo de los padecimientos crónicos, que avanzarán hasta el final de la vida. El desafío, consiste en proporcionar confort a pacientes y familiares, siempre vulnerables por un alto nivel de sufrimiento 
debido a los diferentes síntomas provocados tanto por la evolución de la enfermedad como por los tratamientos. Estos malestares físicos, emocionales y espirituales deben atenderse a lo largo de todo el proceso de la enfermedad hasta que, de manera lamentable, se encuentren fuera de tratamiento oncológico.

Todo padecimiento agobia al paciente y su circunstancia, para ello debemos evaluarlo, al cuidador principal, familia nuclear y al grupo social. En todos los casos se documenta la evaluación con pronóstico y funcionalidad, planificar para organizar su manejo y proyectar alternativas, implementar el tratamiento para control de síntomas y anticipar control de otros posibles eventos, la integración permite ajustarse a la condición socioeconómica y cultural. Durante este acompañamiento se requiere centrar los problemas frecuentes antes de convertirse en dilemas éticos, como en el caso de la aceptación y afrontamiento del diagnóstico y del pronóstico con respeto a la Autonomía y Beneficencia del paciente, o en la posible decisión de la limitación de tratamientos.

Los opioides, indispensables en el manejo de los pacientes con cáncer, se han aplicado a pesar de la crisis mundial en su contra. También se ha logrado la aceptación de la voluntad anticipada y la sedación paliativa, su aplicación es causa de debate social, clínico y legal, pero ha permitido el respeto a la autonomía de los pacientes y el respeto de valores y principios bioéticos.

Dadas las condiciones del Centro de Cáncer del Hospital ABC, la visión de este servicio es hacer posible continuar enseñanza y experiencia, dar a conocer los cuidados paliativos y adecuar el esfuerzo terapéutico para otorgar una atención de excelencia. Proporcionar una atención continua desde el diagnóstico de la enfermedad hasta el final.

En adición y paralelo a ello, la algología es parte básica integral de un Centro de Cáncer, la existencia de la importante clínica del dolor bajo la vigilancia continua encabezada por el Dr. Jorge Guajardo y un valioso equipo es un verdadero agregado integral altamente necesario e indispensable.

\section{Metas del Centro de Cáncer ABC ${ }^{11}$}

Utilizar nuestros programas colectivos de investigación básica, clínica, traslacional de última generación para comprender el cáncer a nivel molecular y genómico, catalizar un enfoque integral y multidisciplinario para el erradicar el cáncer a nivel local, nacional y mundial.

1. Avances terapéuticos que modifiquen el paradigma que resultará en una mayor supervivencia y calidad de vida para nuestros pacientes.

2. Ejecutar de forma proactiva estrategias de prevención y control del cáncer para mitigar el aumento de la incidencia y la mortalidad por cáncer que se prevé para el siglo XXI.

3. Capacitar a la próxima generación de líderes en investigación, atención clínica (Tabla 1).

\section{Liderazgo en la investigación de alto impacto centrada en el paciente ${ }^{12}$}

Dirigir esfuerzo y conocimiento para abordar problemas delicados que preocupan a los pacientes: quimiocerebro, neuropatía, fatiga, estado físico funcional, uso de suplementos para mitigar síntomas y los efectos secundarios relacionados con el cáncer y los tratamientos.

Atención especial al desafío que representa la oncogeriatría en manejo del cáncer y los síntomas.

Ayudar a las personas a vivir mejor con cáncer. Los investigadores se centran en los efectos secundarios de la quimioterapia, los factores biológicos como la inflamación que pueden afectar cualquier tipo de tratamiento del cáncer, la reducción de riesgos y las formas innovadoras de controlar los síntomas.

La excelencia en la atención del cáncer no sólo trata de brindar el mejor tratamiento, sino a su vez el ayudar a las personas a vivir una vida rica y plena mientras enfrentan la enfermedad.

Tabla 1: Programas de investigación.

Programa de investigación
- Biología del cáncer
- Control del cáncer y salud poblacional
- Prevención y supervivencia del cáncer
- Estudios y proyectos en terapias innovadoras y experimentales

\section{Recursos compartidos}

\author{
- Manejo bioespécimen \\ - Bioestadística e informática \\ - Farmacología clínica \\ - Optimización y desarrollo de alianzas potenciales \\ - Estudios mutagénicos
}




\section{Final}

Uno de los objetivos importantes que se marcó en El Centro de Cáncer fue el de conseguir la acreditación como parte integral del Hospital ABC respecto a la calidad asistencial que el Centro ofrece a los pacientes, por la Joint Commission, Órgano Federal de Acreditación de Hospitales de los Estados Unidos de Norte América, esto supone una forma de demostrar y aprobar la calidad asistencial con vigilancia externa y seguimiento calificado, sus metas en investigación y enseñanza. Además del impulso que representa el continuar con atención y organización estricta en los cuidados de pacientes con cáncer que acuden a la institución.

Como calidad de gestión y atención al paciente en momentos de grandes cambios en el abordaje de cáncer, implica enfrentar retos innovadores con la llegada de nuevos medicamentos para lograr alcanzar mejores resultados con mayor supervivencia, determinar los candidatos adecuados y equilibrar los resultados de tumores que llegan con más fuerza en donde hasta ahora no se presentaban grandes avances.

Desde sus principios, el equipo médico del Centro de Cáncer sustenta un compromiso institucional, continuidad en liderazgo, proyección y desarrollo continuo. Siempre alineados en el avance de la investigación traslacional y la evidencia de investigación colaborativa del cáncer.

Los tratamientos son mucho más precisos y personalizados. Los nuevos conocimientos acerca de las bases moleculares, genéticas y celulares del desarrollo y la progresión del cáncer traen consigo nuevas oportunidades de expandir y mejorar capacidades de prevenir, detectar y tratar esta enfermedad, subrayan día a día la importancia del riesgo y la detección para incidir en un diagnóstico temprano. ${ }^{15-18}$

La meta es trabajar en colaboración, científicos y médicos, para generar y aplicar los conocimientos necesarios para lograr que el cáncer sea una enfermedad crónica con el arte integrativo de la oncología de precisión en cada una las disciplinas participantes en el conocimiento y manejo del cáncer.

$$
\text { Mensaje }{ }^{17}
$$

Por último, los descubrimientos respecto a las causas genéticas del cáncer están encontrando mutaciones y cambios epigenéticos que impulsan la aparición y diseminación de cáncer de colon, esófago, cerebro, mama, orofaringe, ovario y próstata, la progresión de leucemias agudas y sarcomas osteogénicos, asi- mismo están vinculando estos cambios genéticos a nuevas opciones terapéuticas y factores predictivos y de riesgo en la selección de los pacientes. A través de estos esfuerzos, logramos nuestra misión: aplicar los descubrimientos científicos dirigidos a mejorar la prevención, detección, tratamiento, supervivencia y la curación del cáncer.

\section{AGRADECIMIENTO ESPECIAL POR SU APORTACIÓN AL ESCRITO}

Sandra Palacios - Central de mezclas; Dolores de la Mata - Radioterapia; Sergio Moreno - Radiocirugía; Margarita Araujo - Cuidados paliativos; Lenny Gallardo - Cáncer genético; Teresa Toussie - Estudios genéticos; Alejandro Zentella - Investigación biología molecular; Ruby Delgado - Unidad de precisión; Lorena López - Funcionamiento centro cáncer; Isabel Aloi - Centro de calidad de vida; Miriam Fastag Círculo de ganadores grupo de apoyo; Jorge Guajardo - Clínica de dolor; Fabian Martínez - Programa de residencia posgrado; Alejandro Noguez - Dirección de residentes; Daniela Shveid - Liderazgo en investigación.

\section{REFERENCIAS}

1. Institute of Medicine (US) Committee on Cancer Control in Low- and Middle-Income Countries. Cancer Control Opportunities in Low- and Middle-Income Countries. Sloan FA, Gelband H, editors. Washington (DC): National Academies Press (US); 2007. doi: 10.17226/11797.

2. NCI-Designation and Accreditation; [Consulted 07 August 2021]; Available in: https://cancer.ucsf.edu/about/designationand-accreditation

3. Gelband H, Jha P, Sankaranarayanan R, Horton S, editors. Cancer: disease control priorities. Third edition (Volume 3). Washington (DC): The International Bank for Reconstruction and Development/The World Bank; 201. doi: 10.1596/978-14648-0349-9.

4. Palacios GSA, Oropeza CR. Uso de herramientas tecnológicas por la seguridad del paciente: experiencia en México de la implementación del sistema Farmis-Oncofarm; Congreso Centroamericano y del Caribe de Ciencias Farmacéuticas, Panamá; 2013.

5. Informática Médica Farmacéutica. [En línea]; [Citado 16 de marzo de 2020] Available in: http://www.imf.es

6. Climente MM, Jiménez TNV. Manual para la atención farmacéutica. 3a ed. Edita: AFAHPE. Hospital Universitario Dr. Peset. Valencia, 2005.

7. Tratamientos contra el cáncer, más allá de la quimioterapia. [En línea]; RDM, València, $24 \bullet 10 \bullet 19$; Available in: https:// www.levante-emv.com/branded-content/2019/10/24/ tratamientos-cancer-quimioterapia-54990350.html

8. Yang I, Udawatta M, Prashant GN, Lagman C, Bloch O, Jensen $\mathrm{R}$ et al. Stereotactic radiosurgery for neurosurgical patients: a historical review and current perspectives. World Neurosurg [Internet]. 2019; 122: 522-531. Available from: https://doi.org/10.1016/j.wneu.2018.10.193 
9. Ganz JC. Stereotactic and radiosurgery concepts in Sweden. Prog Brain Res. 2014; 215: 47-56.

10. Beltrán JQ, Carrillo-Ruiz JD. Neurological functional surgery in Mexico: from pre-columbian cranial surgery to functional neurosurgery in the 21st century. World Neurosurg. 2019; 122: 549-558. Available in: https://doi.org/10.1016/j. wneu.2018.11.165

11. The University of Kansas Cancer Center has been recognized as a National Cancer Institute (NCI)-designated cancer center since 2012; [Cited: 16 March 2020], Available in: https://www. kucancercenter.org/research/transformative-research/ncidesignation

12. UR Awarded $\$ 29$ million for national leadership in cancer control research [In line]; [Cited September 30, 2019]; Available in: https://www.urmc.rochester.edu/news/story/ur-awarded-29million-for-national-leadership-in-cancer-control-research
13. Agur Z, Elishmereni M, Kheifetz Y. Personalizing oncology treatments by predicting drug efficacy, side-effects, and improved therapy: mathematics, statistics, and their integration. Wiley Interdiscip Rev Syst Biol Med. 2014; 6 (3): 239-253.

14. Helmy KY, Patel SA, Nahas GR, Rameshwar P. Cancer immunotherapy: accomplishments to date and future promise. Ther Deliv. 2013; 4 (10): 1307-1320. doi: 10.4155/tde.13.88.

15. Vineis P, Wild CP. Global cancer patterns: causes and prevention. Lancet. 2014; 383 (9916): 549-557.

16. Gopal S, Sharpless NE. Cancer as a global health priority. JAMA. 2021. doi: 10.1001/jama.2021.12778.

17. Gerson S. Director's message [Access August 07, 2021]. Available in: https://case.edu/cancer/about-us/directorsmessage

18. CMABC, Base de datos; Farmis-Oncofarm ${ }^{\circledR}$. 